\title{
Recent results on weakly factorial domains
}

\author{
Gyu Whan Chang ${ }^{1, *}$ \\ ${ }^{1}$ Department of Mathematics Education, Incheon National University, Incheon 22012, \\ Republic of Korea
}

\begin{abstract}
In this paper, we will survey recent results on weakly factorial domains base on the results of $[11,13,14]$. Let $D$ be an integral domain, $X$ be an indeterminate over $D, d \in D, R=D\left[X, \frac{d}{X}\right]$ be a subring of the Laurent polynomial ring $D\left[X, \frac{1}{X}\right], \Gamma$ be a nonzero torsionless commutative cancellative monoid with quotient group $G$, and $D[\Gamma]$ be the semigroup ring of $\Gamma$ over $D$. Among other things, we show that $R$ is a weakly factorial domain if and only if $D$ is a weakly factorial GCD-domain and $d=0, d$ is a unit of $D$ or $d$ is a prime element of $D$. We also show that if $\operatorname{char}(D)=0(\operatorname{resp} ., \operatorname{char}(D)=p>0)$, then $D[\Gamma]$ is a weakly factorial domain if and only if $D$ is a weakly factorial GCD domain, $\Gamma$ is a weakly factorial GCD semigroup, and $G$ is of type $(0,0,0, \ldots)$ (resp., $(0,0,0, \ldots)$ except $p)$.
\end{abstract}

\section{Introduction}

Let $D$ be an integral domain with quotient field $K, X$ be an indeterminate over $D, \Gamma$ be a nonzero torsionless commutative cancellative monoid (written additively) with quotient group $G$, and $D[\Gamma]$ be the semigroup ring of $\Gamma$ over $D$. A nonzero nonunit element $p \in D$ is called a primary element if $p \mid a b$ for each $a, b \in D$ implies that $p \mid a$ or $p \mid b^{n}$ for some positive integer $n$; equivalently, $p D$ is a primary ideal. We say that $D$ is a unique factorization domain (UFD) (resp., weakly factorial domain (WFD)) if each nonzero nonunit of $D$ can be written as a finite product of prime (resp. primary) elements. Clearly, a prime element is primary, and hence a UFD is a WFD. The concept of a WFD was first introduced by Anderson and Mahaney [3]. Chang introduced the notion of weakly factorial semigroups [11] in order to study when $D[\Gamma]$ is a WFD. A nonzero nonunit element $s \in \Gamma$ is primary if, for each $a, b \in \Gamma$, $a+b \in s+\Gamma$ implies that $a \in s+\Gamma$ or $n b \in s+\Gamma$ for some positive integer $n$. It is clear that $s \in \Gamma$ is primary if and only if $s+\Gamma$ is a primary ideal. We say that $\Gamma$ is a weakly factorial semigroup if each nonunit of $\Gamma$ is a finite sum of primary elements.

Chang has worked on WFDs $[6-8,11,13,14]$ and its generalization to rings with zero divisors $[10,12]$. In this paper, among them, we survey the recent results of $[11,13,14]$. In Section 1, for easy reference, we review some definitions and preliminary results on $t$ operations, monoinds, semigroup rings, and weakly Krull domains. Let $d \in D$ and $R=$ $D\left[X, \frac{d}{X}\right]$; so $R$ is a subring of the Laurent polynomial ring $D\left[X, \frac{1}{X}\right]$ containing $D[X]$. In Section 2, we show that $R$ is a weakly factorial domain if and only if $D$ is a weakly factorial GCD-domain and $d=0, d$ is a unit of $D$ or $d$ is a prime element of $D$. We also show that if $D$ is a weakly factorial GCD-domain, $p$ is a prime element of $D$, and $n \geq 2$ is an integer,

\footnotetext{
*e-mail: whan@inu.ac.kr
} 
then $D\left[X, \frac{p^{n}}{X}\right]$ is an almost weakly factorial domain with $C l\left(D\left[X, \frac{p^{n}}{X}\right]\right)=\mathbb{Z}_{n}$, the cyclic group of order $n$. In Section 3, we completely characterize when $D[\Gamma]$ is a WFD. We first study the notion of weakly factorial GCD-semigroups. Then, among other things, we prove that if $\operatorname{char}(D)=0$, then $D[\Gamma]$ is a WFD if and only if $D$ is a weakly factorial GCD domain, $\Gamma$ is a weakly factorial GCD semigroup, and $G$ is of type $(0,0,0, \ldots)$. We also show that if $\operatorname{char}(D)=p>0$, then $D[\Gamma]$ is a WFD if and only if $D$ is a weakly factorial GCD domain, $\Gamma$ is a weakly factorial GCD semigroup, and $G$ is of type $(0,0,0, \ldots)$ except $p$. However, we omit the proofs of the results; so the reader who is interested in the proofs can refer to $[11,13,14]$.

\section{Definitions and preliminary results}

In this section, we review some definitions and preliminary results on the $t$-operations, semigroups, semigroup rings, and weakly Krull domains.

\subsection{The $t$-operations}

Let $D$ be an integral doman with quotient field $K$ and $F(D)$ be the set of nonzero fractional ideals of $D$. For $I \in F(D)$, let $I^{-1}=\{x \in K \mid x I \subseteq D\}$; then $I^{-1} \in F(D)$. Hence, we can define the $v$ - and $t$-operations as follows: $I_{v}=\left(I^{-1}\right)^{-1}$, and $I_{t}=\bigcup\left\{J_{v} \mid J \subseteq I\right.$ and $J \in F(D)$ is finitely generated\}. An $I \in F(D)$ is called a $t$-ideal if $I_{t}=I$. A $t$-ideal of $D$ is a maximal $t$-ideal if it is maximal among proper integral $t$-ideals of $D$. Let $t$-Max $(D)$ be the set of maximal $t$-ideals of $D$. It is well known that $t-\operatorname{Max}(D) \neq \emptyset$ if $D$ is not a field; each ideal in $t-\operatorname{Max}(D)$ is a prime ideal; each prime ideal minimal over a $t$-ideal is a $t$-ideal (which implies that every heightone prime ideal is a $t$-ideal); and $D=\bigcap_{P \in t \text {-Max }(D)} D_{P}$. We say that $D$ is of finite $t$-character if every nonzero nonuint of $D$ is contained in only finitely many maximal $t$-ideals of $D$.

An $I \in F(D)$ is said to be $t$-invertible if $\left(I I^{-1}\right)_{t}=D$. Let $T(D)$ be the group of $t$-invertible fractional $t$-ideals of $D$ under $I * J=(I J)_{t}$, and $\operatorname{Prin}(D)$ be its subgroup of principal fractional ideals. Then $C l(D)=T(D) / \operatorname{Prin}(D)$, called the $t$-class group of $D$, is an abelian group. We say that $D$ is a Prïfer $v$-multiplication domain $(\mathrm{P} v \mathrm{MD})$ if each nonzero finitely generated ideal of $D$ is $t$-invertible. It is known that $D$ is a $\mathrm{P} v \mathrm{MD}$ if and only if $D[X]$ is a $\mathrm{P} v \mathrm{MD}[26$, Corollary 4], if and only if $D\left[X, \frac{1}{X}\right]$ is a PvMD [24, Theorem 3.10]. Also, $D$ is a GCD-domain if and only if $D$ is a $\mathrm{P} v \mathrm{MD}$ with $C l(D)=\{0\}$ [9, Proposition 2].

\subsection{Semigroups}

Let $\Gamma$ be a nonzero torsionless grading monoid, i.e., a nonzero torsionless commutative cancellative monoid (written additively), and $\langle\Gamma\rangle=\{a-b \mid a, b \in \Gamma\}$ be the quotient group of $\Gamma$; so $\langle\Gamma\rangle$ is a torsionfree abelian group and $\Gamma$ is given a total order compatible with the monoid operation [22, page 123]. Let $G$ be a torsionfree abelian group. We say that $G$ is of type $(0,0,0, \ldots)$ if $G$ satisfies the ascending chain condition on its cyclic subgroups (equivalently, for each nonzero element $g \in G$, there exists a largest positive integer $n_{g}$ such that $n_{g} x=g$ is solvable in $G$ ) [17, Theorem 14.10]. For a prime number $p, G$ is said to be of type $(0,0,0, \ldots)$ except $p$ if $G$ satisfies the following two conditions; for each nonzero element $g \in G$, (i) the number of prime numbers dividing $g$ is finite and (ii) for each prime number $q \neq p, q^{n}$ does not divide $g$ for some positive integer $n$. The notion of type $(0,0,0, \ldots)$ except $p$ was introduced by Matsuda [23, 25] in order to study when $K[G]$, where $K$ is a field with $\operatorname{char}(K)=p$, is a generalized Krull domain. Clearly, a torsionfree abelian group of type $(0,0,0, \ldots)$ is of type $(0,0,0, \ldots)$ except $p$ for all prime numbers $p$. As in the case of integral domains, we can define the $t$-operation on $\Gamma$. For more on definitions and basic results (e.g., maximal $t$-ideals, class groups, weakly factorial semigroups), see [20, Chapter 11], [15], or [11]. 


\subsection{Semigroup rings}

For a nonzero torsionless commutative cancellative monoid $\Gamma$ and an integral domain $D$, let $D[\Gamma]$ be the semigroup ring of $\Gamma$ over $D$. Then $D[\Gamma]$ is an integral domain [17, Theorem 8.1] and each nonzero element $f \in D[\Gamma]$ can be written uniquely as $f=a_{1} X^{s_{1}}+\cdots+a_{n} X^{s_{n}}$ for $0 \neq a_{i} \in D$ and $s_{i} \in \Gamma$ with $s_{1}<\cdots<s_{n}$ [17, Corollary 3.4]. Each nonzero element of the form $a X^{s} \in D[\Gamma]$ is said to be homogeneous with $\operatorname{deg}\left(a X^{s}\right)=s$. Let $H$ be the set of nonzero homogeneous elements of $D[\Gamma]$. Then $D[\Gamma]_{H}=K[\langle\Gamma\rangle]$, and hence $D[\Gamma]_{H}$ is a completely integrally closed GCD-domain [18, Theorem 6.4]. For more on semigroup rings, the reader can refer to [17].

\subsection{Weakly Krull domains}

Let $X^{1}(D)$ be the set of height-one prime ideals of an integral domain $D$. We say that $D$ is a weakly Krull domain if $D=\bigcap_{P \in X^{1}(D)} D_{P}$ and this intersection has finite character, i.e., each nonzero nonunit of $D$ is a unit in $D_{P}$ except finitely many primes in $X^{1}(D)$.

Lemma 1.1. $D$ is a weakly Krull domain if and only if $D=\bigcap_{P \in X^{1}(D)} D_{P}$ and $D$ is of finite $t$-character. In this case, $t$ - $\operatorname{dim}(D)=1$, i.e., $t$-Max $(D)=X^{1}(D)$.

Proof. [4, Lemma 2.1].

A nonzero prime ideal $Q$ of $D[X]$ is called an upper to zero in $D[X]$ if $Q \cap D=(0)$, and we say that $D$ is a UMT-domain if each upper to zero in $D[X]$ is a maximal $t$-ideal of $D[X]$. It is known that $D$ is a PvMD if and only if $D$ is an integrally closed UMT-domain [21, Proposition 3.2].

Proposition 1.2. The following statements are equivalent for $D$.

1. $D[X]$ is a weakly Krull domain.

2. $D\left[X, X^{-1}\right]$ is a weakly Krull domain.

3. D is a weakly Krull UMT-domain.

Proof. [2, Propositions 4.7 and 4.11].

Clearly, Krull domains are weakly Krull domains. Recall that $D$ is a UFD if and only if $D$ is a Krull domain and $C l(D)=\{0\}$ [16, Proposition 6.1].

Theorem 1.3. $D$ is a WFD if and only if $D$ is a weakly Krull domain and $C l(D)=\{0\}$.

Proof. [5, Theorem].

An almost weakly factorial domain (AWFD) is an integral domain $D$ in which for each $0 \neq d \in D$, there is an integer $n=n(d) \geq 1$ such that $d^{n}$ can be written as a finite product of primary elements of $D$. It is known that $D$ is an AWFD if and only if $D$ is a weakly Krull domain with $C l(D)$ torsion [4, Theorem 3.4]; hence

$$
\mathrm{UFD} \Rightarrow \mathrm{WFD} \Rightarrow \mathrm{AWFD} \Rightarrow \text { weakly Krull domain. }
$$

It is easy to see that if $N$ is a multiplicative subset of a weakly Krull domain (resp., WFD, an AWFD), then $D_{N}$ satisfies the corresponding property. 


\section{Weakly factorial generalized Rees rings}

Let $D$ be an integral domain, $I$ be a proper ideal of $D$, and $t$ be an indeterminate over $D$. Then $R=D\left[t I, t^{-1}\right]$ is a subring of $D\left[t, t^{-1}\right]$, called the generalized Rees ring of $D$ with respect to $I$. In [27], Whithman proved that if $I$ is finitely generated, then $R$ is a UFD if and only if $D$ is a UFD and $t^{-1}$ is a prime element of $R$. Let $I=d D$ for $d \in D$ and $t^{-1}=X$; so $R=D\left[X, \frac{d}{X}\right]$. In [1], the authors studied several kinds of divisibility properties of $R$ including Krull domains, UFDs, and GCD-domains. In this section we study when $R=D\left[X, \frac{d}{X}\right]$ is a WFD.

Proposition 2.1. Let $d \in D$ be a nonzero element and $R=D\left[X, \frac{d}{X}\right]$. Then the following statements are equivalent.

1. $X$ is irreducible (resp., prime, primary) in $R$.

2. $\frac{d}{X}$ is irreducible (resp., prime, primary) in $R$.

3. $d$ is a nonunit (resp., prime, primary) in D.

Proof. For the properties of irreducible and prime, see [1, Proposition 1]. For the property of primary, see [13, Proposition 2].

Let $0 \neq a \in D$. It is easy to see that $a$ is primary if and only if $\sqrt{a D}$ is a maximal $t$-ideal [7, Lemma 2.1]. Hence, by Proposition 2.1, we have

Corollary 2.2. [13, Corollary 3] Let $d \in D$ be a nonzero nonunit and $R=D\left[X, \frac{d}{X}\right]$. If $d D$ is primary in $D$, then $\sqrt{X R}$ is a maximal $t$-ideal of $R$ and $\left(X, \frac{d}{X}\right)_{v}=R$.

The next lemma can be easily proved by Proposition 1.2 which is necessary for the proof of Thorem 2.4.

Lemma 2.3. [13, Proposition 4] Let $d \in D$ be a nonzero nonunit and $R=D\left[X, \frac{d}{X}\right]$. Then $R$ is a weakly Krull domain if and only if $D$ is a weakly Krull UMT-domain.

By using the results of Corollary 2.2 and Lemma 2.3, we can completely characterize when $R=D\left[X, \frac{d}{X}\right]$ is a WFD.

Theorem 2.4. [13, Theorem 6] Let $d \in D$ and $R=D\left[X, \frac{d}{X}\right]$. Then the following statements are equivalent.

1. $R$ is a WFD.

2. $R$ is a weakly factorial GCD-domain.

3. $D$ is a weakly factorial $G C D$-domain and $d=0, d$ is a unit of $D$, or $d$ is a prime element of $D$.

A ring of Krull type is a $\mathrm{P} v \mathrm{MD}$ of finite $t$-character [19, Theorem 7]. It is known that $D$ is a ring of Krull type if and only if $D[X]$ is a ring of Krull type, or equivalently, $D\left[X, \frac{1}{X}\right]$ is a ring of Krull type (cf. [19, Propositions 9 and 12]).

Theorem 2.5. [13, Theorem 7] Let $d \in D$ and $R=D\left[X, \frac{d}{X}\right]$. Then $R$ is a ring of Krull type if and only if $D$ is a ring of Krull type.

A generalized Krull domain is an integral domain $D$ such that (i) $D_{P}$ is a valuation domain for all $P \in X^{1}(D)$, (ii) $D=\bigcap_{P \in X^{1}(D)} D_{P}$, and (iii) this intersection has finite character. Clearly, $D$ is a generalized Krull domain if and only if $D$ is a weakly Krull P $v$ MD, if and only if $D$ is a ring of Krull type with $t-\operatorname{dim}(D)=1$. Hence, by Lemma 2.3 andTheorem 2.5, we have 
Corollary 2.6. [13, Corollary 8] Let $d \in D$ and $R=D\left[X, \frac{d}{X}\right]$. Then $R$ is a generalized Krull domain if and only if $D$ is a generalized Krull domain.

It is known that if $D$ is a UFD, $p$ is a prime element of $D$, and $n \geq 1$ is an integer, then $D\left[X, \frac{p^{n}}{X}\right]$ is a Krull domain with $C l\left(D\left[X, \frac{p^{n}}{X}\right]\right)=\mathbb{Z}_{n}[1$, Theorems 8 and 16]. The next result is an AWFD analog.

Corollary 2.7. [13, Corollary 9] Let D be a weakly factorial GCD-domain, $p$ be a prime element of $D$, and $n \geq 2$ be an integer. Then $R=D\left[X, \frac{p^{n}}{X}\right]$ is an AWFD with $C l(R)=\mathbb{Z}_{n}$.

Let $V$ be a rank-one nondiscrete valuation domain, $y$ be an indeterminate over $V$, and $D=V[y]$. Then $D$ is a weakly factorial GCD-domain and $y$ is a prime of $D$. Thus, by Theorem 2.4 and Corollary 2.7, $R=D\left[X, \frac{y}{X}\right]$ is a WFD and $D\left[X, \frac{y^{n}}{X}\right]$ is an AWFD with $C l\left(D\left[X, \frac{y^{n}}{X}\right]\right)=\mathbb{Z}_{n}$ for all integers $n \geq 2$.

\section{Weakly factorial semigroup rings}

Let $D$ be an integral domain with quotient field $K, \Gamma$ be a torsionless grading monoid with quotient group $G, D[\Gamma]$ be the semigroup ring of $\Gamma$ over $D$, and $H$ be the set of nonzero homgeneous elements of $D[\Gamma]$.

In order to study when $D[\Gamma]$ is a UFD, we first need the notion of factorial semigroups. As in the domain case, an $\alpha \in \Gamma$ is called a prime element if $\alpha+\Gamma$ is a prime ideal of $\Gamma$, and we say that $\Gamma$ is a factorial semigroup if each nonunit of $\Gamma$ can be written as a finite sum of prime elements of $\Gamma$.

Theorem 3.1. $D[\Gamma]$ is a UFD if and only if $D$ is a UFD, $\Gamma$ is a factorial semigroup, and the quotient group of $\Gamma$ is of type $(0,0,0, \ldots)$.

Proof. [18, Theorem 7.17 and Lemma 7.15].

Theorem 3.1 is the motivation of the results in this section, i.e., we completely characterize when $D[\Gamma]$ is a WFD.

\subsection{Weakly factorial semigroups}

Lemma 3.2. [11, Lemma 1] Let $\alpha \in \Gamma$ be a nonunit. Then the following statements are equivalent.

1. $X^{\alpha}$ is primary in $D[\Gamma]$.

2. $\sqrt{X^{\alpha} D[\Gamma]}$ is a maximal $t$-ideal of $D[\Gamma]$.

3. $D[\sqrt{\alpha+\Gamma}]$ is a maximal $t$-ideal of $D[\Gamma]$.

4. $\sqrt{\alpha+\Gamma}$ is a maximal $t$-ideal of $\Gamma$.

5. $\alpha$ is primary in $\Gamma$.

It is well known that $D$ is a GCD-domain if and only if $a D \cap b D$ is principal, if and only if $(a, b)_{v}$ is principal for each pair $0 \neq a, b \in D$. Similarly, note that if $\Gamma$ is a GCD-semigrup, then $\operatorname{gcd}(\alpha, \beta)=a \Leftrightarrow(\alpha+\Gamma) \cap(\beta+\Gamma)=\alpha+\beta-a+\Gamma, \Leftrightarrow((\alpha+\Gamma) \cup(\beta+\Gamma))_{v}=a+\Gamma$ for each pair $\alpha, \beta \in \Gamma$. Thus, $\Gamma$ is a GCD-semigroup if and only if $\bigcap_{i=1}^{k}\left(\alpha_{i}+\Gamma\right)$ is principal, if and only if $\left(\bigcup_{i=1}^{k}\left(\alpha_{i}+\Gamma\right)\right)_{v}$ is principal for any $\alpha_{1}, \ldots, \alpha_{k} \in \Gamma$ (cf. [20, Exercise 1 (page 117) and Theorem 11.5]). The next result is the semigroup analog of [3, Theorem 18] that a WFD $D$ is a GCD-domain if and only if $D_{P}$ is a valuation domain for each height-one prime ideal $P$ of $D$. 
Proposition 3.3. [11, Proposition 5] Suppose that $\Gamma$ is a weakly factorial semigroup. Then $\Gamma$ is a GCD-semigroup if and only if $\alpha+\Gamma \subseteq \beta+\Gamma$ or $\beta+\Gamma \subseteq \alpha+\Gamma$ for all primary elements $\alpha$ and $\beta$ of $\Gamma$ with $\sqrt{\alpha+\Gamma}=\sqrt{\beta+\Gamma}$.

It is clear that a factorial semigroup is a GCD-semigroup. Also, prime elements are primary, and hence a factorial semigroup is a weakly factorial GCD-semigroup. However, the next example shows that a weakly factorial GCD-semigroup whose quotient group is of type $(0,0,0, \ldots)$ need not be a factorial semigroup.

Example 3.4. [11, Example 12] Let $G=\{m+n \sqrt{2} \mid m, n \in \mathbb{Z}\}$ be the additive group and $\Gamma=\{\alpha \in G \mid \alpha \geq 0\}$ be a subsemigroup of $G$.

1. $\Gamma$ is a torsionless grading monoid with quotient group $G$.

2. $G$ is of type $(0,0,0, \ldots)$.

3. $\Gamma$ is a weakly factorial GCD-semigroup.

4. $\Gamma$ is not a factorial semigroup.

Proof. (1), (2) and (3). These are easy exercises. (4) This can be proved by the fact that $\sqrt{2}$ is irrational.

\subsection{When $G$ is of type $(0,0,0, \ldots)$}

Let $D[X]$ be the polynomial ring over $D$ and $\mathbb{N}_{0}$ be the semigroup of nonnegative integers. Then (i) $D[X]=D\left[\mathbb{N}_{0}\right]$ and (ii) $D[X]$ is a WFD if and only if $D$ is a weakly factorial GCDdomain (Theorem 2.4). Note that $\mathbb{N}_{0}$ is a torsionless grading monoid whose quotient group $\mathbb{Z}$ is of type $(0,0,0, \ldots)$; hence the next result is a natural generalization of the result (ii) to $D[\Gamma]$ with $G$ of type $(0,0,0, \ldots)$.

Theorem 3.5. [11, Theorem 9] Assume that $G$ is of type $(0,0,0, \ldots)$. Then $D[\Gamma]$ is a WFD if and only if $D$ is a weakly factorial GCD-domain and $\Gamma$ is a weakly factorial GCD-semigroup.

Since a factorial semigroup is a weakly factorial GCD semigroup, by Theorem 3.5, we have

Corollary 3.6. [11, Corollary 10] Assume that $G$ is of type $(0,0,0, \ldots)$ and $\Gamma$ is a factorial semigroup. Then $D[\Gamma]$ is a WFD if and only if $D$ is a weakly factorial GCD-domain.

Set $H_{\alpha}=\mathbb{N}_{0}$ for each $\alpha$, and let $\Gamma=\sum_{\alpha} H_{\alpha}$. Then $K[\Gamma]=K\left[\left\{X_{\alpha}\right\}\right]$ is a factorial domain, and hence $\Gamma$ is a factorial semigroup whose quotient group is of type $(0,0,0, \ldots)$. Thus, by Corollary 3.6, we have

Corollary 3.7. [11, Corollary 11] Let $\left\{X_{\alpha}\right\}$ be a nonempty set of indeterminates over D. Then $D\left[\left\{X_{\alpha}\right\}\right]$ is a WFD if and only if $D$ is a weakly factorial GCD-domain.

Example 3.8. Let $\mathbb{Z}$ be the ring of integers and $\Gamma=\{m+n \sqrt{2} \mid m, n \in \mathbb{Z}$ and $m+n \sqrt{2} \geq 0\}$. Then $\mathbb{Z}[\Gamma]$ is a WFD but not a UFD by Theorems 3.1, 3.5 and Example 3.4.

The next example shows that Theorem 3.5 is not true when $G$ is not of type $(0,0,0, \ldots)$.

Example 3.9. [11, Example 13] Let $\mathbb{R}$ be the field of real numbers and $\Gamma$ be the additive semigroup of nonnegative rational numbers.

1. $\Gamma$ is a torsion-free grading monoid. 
2. $\Gamma$ is a weakly factorial GCD-semigroup.

3. $\mathbb{R}[\Gamma]$ has the (Krull) dimension one, and hence $t-\operatorname{dim}(\mathbb{R}[\Gamma])=1$.

4. $\mathbb{R}[\Gamma]$ is not a WFD.

Proof. (1) Clear. (2) This is an easy exercise. (3) Let $\mathbb{N}_{0}$ be the semigroup of nonnegative integers. Then $\mathbb{R}[\Gamma]$ is integral over $\mathbb{R}\left[\mathbb{N}_{0}\right]$ and $\operatorname{dim}\left(\mathbb{R}\left[\mathbb{N}_{0}\right]\right)=1$. Thus, $\operatorname{dim}(\mathbb{R}[\Gamma])=t$ $\operatorname{dim}(\mathbb{R}[\Gamma])=1$. (4) It can be shown that $1-X \in \mathbb{R}[\Gamma]$ is contained in infinitely many height-one prime ideals of $\mathbb{R}[\Gamma]$.

\section{$3.3 \operatorname{char}(D)=0$}

In this section, we study the WFD property of $D[\Gamma]$ under the assumption that $\operatorname{char}(D)=0$. It is known that if $\operatorname{char}(D)=0$, then $D[G]$ is a ring of Krull type if and only if $D$ is a ring of Krull type and $G$ is of type $(0,0,0, \ldots)$ [25, Theorem 3].

Lemma 3.10. [14, Theorem 3.1] Let $G$ be a torsionfree abelian group, $D[G]$ be the group ring of $G$ over $D$, and assume that char $(D)=0$. Then $D[G]$ is of finite t-character if and only if $D$ is of finite $t$-character and $G$ is of type $(0,0,0, \ldots)$.

Corollary 3.11. [14, Corollary 3.2] Let $K$ be a field, $G$ be a torsionfree abelian group, and assume that $\operatorname{ch}(K)=0$. Then the following statements are equivalent.

1. $K[G]$ is a WFD.

2. $K[G]$ is a weakly Krull domain.

3. $K[G]$ is of finite $t$-character.

4. $G$ is of type $(0,0,0 \ldots)$.

5. $K[G]$ is a $U F D$.

6. $K[G]$ is a Krull domain.

7. $K[G]$ is a generalized Krull domain.

Proof. (1) $\Rightarrow$ (2) $\Rightarrow$ (3) Clear. (3) $\Rightarrow$ (4) Lemma 3.10. (4) $\Rightarrow$ (5) Theorem 3.1. (5) $\Rightarrow$ (1) Clear. (5) $\Rightarrow(6) \Rightarrow(7) \Rightarrow$ (3) Clear.

Theorem 3.12. [14, Theorem 3.4] Assume that char $(D)=0$. Then $D[\Gamma]$ is a WFD if and only if $D$ is a weakly factorial GCD-domain, $\Gamma$ is a weakly factorial GCD-semigroup, and $G$ is of type $(0,0,0, \ldots)$.

Proof. If $D[\Gamma]$ is a WFD, then $D[\Gamma]_{H}$ is a WFD. Note that $D[\Gamma]_{H}=K[G]$; hence $G$ is of type $(0,0,0 \ldots)$ by Corollary 3.11 . Thus, the result follows directly from Theorem 3.5 .

\section{$3.4 \operatorname{char}(D)=p>0$}

In this section, we study when $D[\Gamma]$ is a WFD under the assumption that $\operatorname{char}(D)=p>0$. Recall that if $\operatorname{char}(D)=p>0$, then $D[G]$ is a ring of Krull type if and only if $D$ is a ring of Krull type and $G$ is of type $(0,0,0, \ldots)$ except $p[25$, Theorem 5]. 
Lemma 3.13. [14, Theorem 4.2] Let $G$ be a torsionfree abelian group, $D[G]$ be the group ring of $G$ over $D$, and assume that char $(D)=p>0$. Then $D[G]$ is of finite $t$-character if and only if $D$ is of finite $t$-character and $G$ is of type $(0,0,0, \ldots)$ except $p$.

Corollary 3.14. [14, Corollary 4.3] Let $K$ be a field, $G$ be a torsionfree abelian group, and assume that $\operatorname{char}(K)=p>0$. Then the following statements are equivalent.

1. $K[G]$ is a WFD.

2. $K[G]$ is a weakly Krull domain.

3. $K[G]$ is of finite $t$-character.

4. $G$ is of type $(0,0,0 \ldots)$ except $p$.

5. $K[G]$ is a generalized Krull domain.

We next give a complete characterization of a WFD $D[\Gamma]$ with $\operatorname{char}(D)=p>0$.

Theorem 3.15. [14, Theorem 4.5] Assume that char $(D)=p>0$. Then $D[\Gamma]$ is a WFD if and only if $D$ is a weakly factorial GCD-domain, $\Gamma$ is a weakly factorial GCD-semigroup, and $G$ is of type $(0,0,0, \ldots)$ except $p$.

Example 3.16. [14, Example 4.6] Let $K$ be a field, $p$ be a prime number, $G=\bigcup_{n=1}^{\infty}\left(1 / p^{n}\right) \mathbb{Z}$, and $K[G]$ be the group ring of $G$ over $K$.

1. $G$ is of type $(0,0,0 \ldots)$ except $p$ but not of type $(0,0,0 \ldots)$.

2. If $\operatorname{char}(K)=p>0$, then $K[G]$ is a weakly factorial domain.

3. If $\operatorname{char}(K) \neq p$ (e.g., $\operatorname{char}(K)=0)$, then $K[G]$ is not a WFD.

Hence, $\mathbb{R}[G]$ is not a WFD, while $\mathbb{Z}_{p}[G]$ is a WFD, where $\mathbb{Z}_{p}$ is a field of $p$ elements.

Proof. For (1), see [25, Proposition 12]. For (2) and (3), see (1) and Theorems 3.5 and 3.15 .

\section{References}

[1] D.D. Anderson and D.F. Anderson, The ring $R[X, r / X]$, in: Zero-Dimensional Commutative Rings, in: Lecture Notes in Pure and Appl. Math., vol. 171, (Marcel Dekker, New York, 1995), pp. 95-113.

[2] D.D. Anderson, E. Houston, and M. Zafrullah, t-linked extensions, the t-class group, and Nagata's theorem, J. Pure Appl. Algebra 86, 109-124 (1993).

[3] D.D. Anderson and L.A. Mahaney, On primary factorizations, J. Pure Appl. Algebra 54, 141-154 (1988).

[4] D.D. Anderson, J. Mott, and M. Zafrullah, Finite character representations for integral domains, Bollettino U.M.I. 6-B(7), 613-630 (1992).

[5] D.D. Anderson and M. Zafrullah, Weakly factorial domains and groups of divisiblity, Proc. Amer. Math. Soc. 109, 907-913 (1990).

[6] D.F. Anderson and G.W. Chang, Homogeneous splitiing sets of a graded integral domain, J. Algebra 288, 527-544 (2005).

[7] D.F. Anderson, G.W. Chang, and J. Park, Generalized weakly factorial domains, Houston J. Math. 29, 1-13 (2003). 
[8] D.F. Anderson, G.W. Chang, J. Park, Weakly Krull and related domains of the form $D+M$, $A+X B[X]$, and $A+X^{2} B[X]$, Rocky Mountain J. Math. 36, 1-22 (2006).

[9] A. Bouvier, Le groupe des classes d'un anneau intégré, 107ème Congrès National des Sociétés Savantes, Brest, fasc. IV, 85-92 (1982).

[10] G.W. Chang, Weakly factorial rings with zero divisors, in: Ideal Theoretic Methods in Commutative Algebra, in: Lecture Notes in Pure and Appl. Math., vol. 220, (Marcle Dekker, New York, 2001), pp. 119-131.

[11] G.W. Chang, Semigroup rings as wekly factoral domains, Comm. Algebra 37, 32783287 (2009).

[12] G.W. Chang, Some examples of weakly factorial rings, Korean J. Math. 21, 319-323 (2013).

[13] G.W. Chang, Weakly factorial property of a generalized Rees ring $D\left[X, \frac{d}{X}\right]$, Rocky Mountain J. Math., to appear.

[14] G.W. Chang and D.Y. Oh, Semigroup rings as wekly factoral domains, II, Preprint.

[15] S. El Baghdadi, L. Izelgue, and S. Kabbaj, On the class group of a graded domain, J. Pure Appl. Algebra 171, 171-184 (2002).

[16] R. Fossum, The Divisor Class Group of a Krull Domain, (Springer, New York, 1973).

[17] R. Gilmer, Commutative Semigroup Rings, (The Univ. of Chicago Press, Chicago, 1984).

[18] R. Gilmer and T. Parker, Divisiblility properties in semigroup rings, Michigan Math. J. 21, 65-86 (1974).

[19] M. Griffin, Some results on v-multiplication rings, Canad. Math. J. 19, 710-722 (1967).

[20] F. Halter-Koch, Ideal System: An introduction to Multiplicative Ideal Theory, (Marcel Dekker, New York, 1998).

[21] E. Houston and M. Zafrullah, On t-invertibility II, Comm. Algebra 17(8), 1955-1969 (1989).

[22] D.G. Northcott, Lessons on Rings, Modules, and Multiplicities, (Cambridge Univ. Press, Cambridge, 1968).

[23] R. Matsuda, Torsion-free abelian semigroup rings III, Bull. Fac. Sci. Ibaraki Univ. 9, 1-49 (1977).

[24] R. Matsuda, Torsion-free abelian semigroup rings IV, Bull. Fac. Sci. Ibaraki Univ. Ser. A 10, 1-27 (1978).

[25] R. Matsuda, Krull properties of semigroup rings, Bull. Fac. Sci. Ibaraki Univ. 14, 1-12 (1982).

[26] H. Uda, A characterization of Prüfer v-multiplication domains in terms of polynomial grade, Hiroshima Math. J. 16, 115-120 (1986).

[27] D. Whitman, A note on unique factorization in Rees rings, Math. Japon. 17, 13-14 (1972). 